\title{
Chemical and Different Nutritional Characteristics of Brown Seaweed Lipids
}

\author{
Niaz Ahmed*, Kashif Ahmed \\ Department of Chemistry, N.E.D. University of Engineering \& Technology, Karachi, 75270, Pakistan
}

A R T I C L E I N F O

Article history:

Received: 20 March, 2016

Accepted: 25 April, 2016

Online: 25 April, 2016

Keywords:

Brown seaweed lipids

Eicosapentaenoic acid

Fucoxanthin

Glycoglycerolipids

Oxidative stability

Stearidonic acid

\section{Introduction}

Algae can be divided into two main groups, macroalgae (seaweeds) and micro-algae. Approximately 9000 seaweed species are available worldwide, which are further divided into three main groups on the basis of their pigmentation: brown (Phaeophyta), red (Rhodophyta) and green (Chlorophyta) seaweeds. Whole parts of seaweed are available for effective biomass sources because of their high rate of absorption of carbon dioxide from the atmosphere.

Seaweeds have been used as sources of food, medicine, cosmetics, fertilizer, feed and bio-energy [1-3]. Seaweeds are enriched in non-starch polysaccharides food components such as carrageenan and alginate, which cannot be degraded by mammalian enzymes [4]. These contain high content of amino acid then the other vegetable. These are also enriched in polyphenols, especially phlorotannins which is used as an antioxidant. Although lipid content in seaweed is lower than marine fish but due to their large stock in coastal waters define it as potential source of functional lipid. Lipid content in oily fish has been reported to be approximately $20 \mathrm{wt}$. \% per dry weight (DW), occasionally reaching 50 wt. \% per DW, but seaweeds contain up to $1-5$ wt. $\%$ total lipids per DW. $[5,6]$. On the other hand, recent research revealed that contents of TL and omega-3 polyunsaturated fatty acids of seaweeds fluctuate seasonally, it could reach $15 \%$ TL per DW and could contain over $40 \%$ omega-3 PUFAs per total fatty acids.

Several types of bioactive compounds, such as omega3 PUFAs, omega-6 arachidonic acid, fucoxanthin, fucosterol and some polyphenols are present in brown seaweeds. Carotenoid

"Corresponding Author: Niaz Ahmad, Email: niazahmed29732@gmail.com which is found in brown seaweeds shows several physiological effects due to their unique molecular mechanisms [7].

\section{Seaweed lipids}

The lipid content in seaweed is very sensitive and remarkably change by species to species, it also varies by geographically, seasonally, temperature, salinity and light intensity [8].

A latest research revealed that species of tropical areas have significantly Lower lipid contents than the cold area species. A quantitative lipid analysis showed that the TL content in Sargassaceae (a major brown seaweed family) was higher in subarctic zones (approximately $6 \%$ per DW) than tropical zones (0.9-1.9\% per DW) [9, 10].

\section{Brown seaweed lipids}

Seaweeds play a vital role in preserving coastal belt ecosystems especially brown seaweeds $[11,12]$. In Japan brown seaweeds are form a part of the staple diet. They contain valuable nutrients and bioactive components, which is not found in terrestrial plants. Major membrane lipid is also present in brown seaweeds. The composition of fatty acid of the chloroplast Glycoglycerol lipids is rich in highly unsaturated fatty acid. The main fatty acid in the GL of plant leaf is alfa linolenic acid (LNA), which is rich in monogalactosyl-diacylglycerols (MGDG), digalactosyl-diacylglycerols (DGDG), Stearidonic acid (SDA), arachidonic acid (ARA) and eicosapentaenoic acid (EPA). These are the major fatty acids in seaweeds [13, 14]. A clinical study expresses about the omega-3 PUFAs that it has important cardio-protective effect [15] and the reduction of cardiovascular disease (CVD) occur by taking of EPA and 
docosahexaenoic acid (DHA) [16,17]. ARA, the active form of omega-6 PUFA, is found help ful to develop immune response, thrombosis and brain function. ARA and DHA are also useful for neuro development of infant so it should be used in diet supplement. ARA and DHA are major constituents of cell membranes and play an important role in the structure of neurons in the central nervous system, where these are present at high concentrations [18]. Humans have a poor ability to form DHA from LNA or ARA from linoleic acid (LA) due to the low activity of delta-6 desaturase. Brown seaweed lipids contain fucosterol and fucoxanthin as functional components. Fucoxanthin exhibits characteristic biological activity, including anti-obesity [19], anti-diabetic, antioxidant and anti-cancer effects. The physiological effects of fucoxanthin have been described in detail in several reviews [20,21].

\section{Seaweed lipids Oxidative stability}

Due to large number of double bonds, the major PUFAs in brown seaweed lipids, such as SDA are very easily oxidized $[22,23]$. Oxidative deterioration of these PUFAs is one of the very important problems in food chemistry, as lipid oxidation produce undesirable flavors, odor and lower nutritional quality and storage of lipid-containing foods. Carotenoids are degraded by carotenoid-radical inter actions and cleavage at the center of the carotenoid bone. The electron-rich status of fucoxanthin makes it less stable than other carotenoids. Thus, the low stability of fucoxanthin may be a major problem in the application of brown seaweeds to food materials as fucoxanthin resources.

\section{High oxidative stability of PUFAs as GL form}

GL plays an important role in photosynthesis. It is very rich in omega-3 PUFAs such as SDA, EPA and LNA. The content of these PUFAs increases with the increase of photosynthetic activities [24]. The GL of chloroplast is always exposed to oxidative stress because it has high level of PUFAs and light energy absorption for photosynthesis. Reactive oxygen species (ROS) are developed in the photosynthetic membrane. This can cause oxidative damage to many cellular components, including proteins, lipids membrane, nucleic, chlorophyll and nucleic acids. To maintain the level of ROS and to save the cells, seaweeds possess a number of antioxidants (phenolic , ascorbate, glutathione compounds, carotenoids and tocopherols) and enzymes (Catalase, Superoxide dismutase , Glutathione reductase and Ascorbate peroxidase) to scavenge the ROS and to regenerate the active forms of antioxidants $[25,26]$. A latest research showed that PUFAs in GL form has high oxidative stability [27]. GL also showed higher oxidative stability than Soybean oil. It is due to the protective effect of sulfoquinovosyl and galactosyl moieties on PUFAs bonded to the same GL molecule. If we want to decrease in the oxidative stability of food lipids we should also use carbohydrates [28].

\section{Brown seaweed lipids Nutritional impact}

Brown seaweed lipids have up to $5 \%$ fucoxanthin [10]. Fucoxanthin shows Anti-obesity effects [19]. It also improves insulin resistance and remarkably decreases blood glucose level [29]. A research shows that when brown seaweed lipids are given to obesity/ diabetes model mice, excess fat accumulation in abdominal white adipose tissue (WAT) is gradually reduced, and glucose levels are again restored to normalize levels, mainly due to presence of fucoxanthin in the lipids.

It is also observed that a combination diet of $0.1 \%$ fucoxanthin and fish oil also markedly decrease the blood glucose and plasma insulin quantity to the same levels. But omega-3 fish oil alone had very little effect on Abdominal WAT weight and plasma glucose level, as brown seaweed lipids contain high levels of omega-3 PUFAs, so the effect of fucoxanthin will be greater compared with that fucoxanthin alone. Another research shows that when brown coloured seaweed lipids were given to Animals, a significant increase in the component of DHA and ARA of the liver was also observed [7].

\section{Conclusion}

The brown seaweed families Laminariales and Fucales are two basic species, forming underwater forests which provide and develop different ecological services and effect to coastal belt ecosystems. They are also a major class of aqua cultured seaweeds and are regarded as having significant potential to serve as a biomass source. The brown seaweeds contain polysaccharides, including undigested fibers, minerals, proteins and lipids. Although the lipid content of brown seaweeds is less than the content of other nutrients, it contains biologically active compounds, such as fucoxanthin, omega-3 EPA and SDA and omega-6 ARA. Among these compounds, fucoxanthin is major to understand the characteristic functionality of brown seaweed lipids. The oxidative stability of PUFAs is occasionally problematic in the application of marine lipids to food and other products. A research study indicated the high oxidative stability of omega-3 PUFAs in brown seaweed lipids. Although more research study will be needed to confirm this characteristic oxidative stability of PUFAs in brown seaweed lipids, these lipids may be applied to nutraceuticals and functional foods as an oxidative stable omega-3 source. For the commercial use of brown seaweed lipids, a search for TL-rich seaweed materials will be important. Studies on the seasonal, regional and species variations of lipid components showed that several brown seaweed species collected in the growing stage had high levels of TL, fucoxanthin and omega-3 PUFAs.

\section{Conflict of interest}

The authors declare that there is no conflict of interests regarding the publication of this paper.

\section{References}

[1] Jang S-S, Shirai Y, Uchida M and Wakisaka M, "Production of mono sugar from acid hydrolysis of seaweed," Afric. J. Biotec., 11, 1953-1963, 2012.

[2] Khan W, Rayirath U. P, Subramanian S, Jithesh M. N, Rayorath P, Hodges D. M, ritchley A. T, Craigie J. S , Norrie J and Prithiviraj B "Seaweed extracts as biostimulants of plant growth and development," J. Plant Growth Regu., 28, 386-399, 2009.

[3] Pangestuti R, Kim S-K, "Biological activities and health benefit effects of natural pigments derived from marine algae," J. Func. Foods, 3, 255-266, 2011.

[4] Wong K. H, Cheung P.C. K "Effect of fiber-rich brown seaweeds on protein bioavailability of casein in growing rats," Int. J. Food Sci. Nut., 54, 269-279, 2003. 
[5] T Arao, Yamada M "Positional distribution of fatty acids in galactolipids of algae," J. Phytochem., 28, 805-810, 1989.

[6] Vaskovsky V. E, Khotimchenko S. V, Xia B, Hefang L "Polar lipids and fatty acid of some marine macrophytes from the Yellow sea," J. Phytochem., 42, 1347-1356, 1996.

[7] Airanthi M. K. W. A, Sasak N, Iwasaki S, Baba N, Abe M, Hosokawa M, Miyashita K.,"Effect of brown seaweed lipids on fatty acid composition and lipid hydro peroxide levels of mouse liver," J. Agric. Food Chem., 59, 4156-4163, 2011

[8] Sa'nchez-Machado D. I, Lo' pez-Cervantes J, Lo'pez-Hernandez J, Paseiro-Losada P "Fatty acids, total lipid, protein and ash contents of processed edible seaweeds," J. Food Chem., 85, 439-444, 2004.

[9] Bhaskar N, Hosokawa M, Miyashita K "Comparitive evaluation of fatty acid composition of different Sargassum (Fucales, Phaeophyta) species harvested fromtemperate and tropicalwaters," J. Aquatic Food Product Techno., 13, 53-70, 2004.

[10] Terasaki M, Hirose A, Narayan B, Baba Y, Kawagoe C, Yasui H, Saga N, Hosokawa M, Miyashita K "Evaluation of recoverable functional lipid components with special reference to fucoxanthin and fucosterol contents of several brown seaweeds of Japan," J. Phycology., 4, 974-980, 2009.

[11] Schmidt A. L, Coll M, Romanuk T. N, Lotze H. K "Ecosystemstructure and services in eelgrass Zosteramarina and rockweed Ascophyllum nodosum habitats," J. Marine Eco. Prog. Series, 437, 51-68, 2011.

[12] Seeley R. H, Schlesinger W. H "Sustainable seaweed cutting? The rockweed (Ascophyllum nodosum) industry of marine and the maritime provinces," 'Annals of the New York Academy of Sciences, 1249, 84-103, 2012.

[13] Harwood J. L, Jones A. L. "Lipid metabolism in algae," J. Advan., Botanical Res., 16, 1-53, 1989.

[14] Smith K. L \& Harwood J. L "Lipids and lipid metabolism in the brown alga, Fucus serratus," J. Phytochem., 23, 2469-2473, 1984.

[15] Russo G. L "Dietary n 6 and n 3 polyunsaturated fatty acids: From biochemistry to clinical implications in cardiovascular prevention," J. Biochem. Pharma., 77, 937-946, 2009.

[16] Kris-Etherton P. M, Harris W. S, Appel L. J "Fish consumption, fish oil, omega-3 fatty acids, and cardiovascular disease," Circulation, 106(21), 2747-2757, 2002.

[17] Leaf A, Kang J. X, Xiao Y-F "Fish oil fatty acids as cardiovascular drugs," J. Curr. Vascular Pharma., 6, 1-12, 2008.

[18] Davis-Bruno K, Tassinari M. S "Essential fatty acid supplementation of DHA and ARA and effects on neurodevelopment across animal species," A review of the literature. Birth Defects Research (Part B) 92, 240-250, 2011.

[19] Maeda H, Hosokawa M, Sashima T, Funayama K, Myashita K "Fucoxanthin from edible seaweed, Undaria pinnatifida, shows antiobesity effect through UCP1 expression in white adipose tissues," J. Biochem. Biophy. Res. Comm., 332(2), 392-397, 2005.

[20] Mohamed S, Hashim S. N, Rahman H. A., "Seaweeds: A sustainable functional food for complementary and alternative therapy," J. Trends Food Sci. \& Techno., 23, 83-96, 2012.

[21] Peng J, Yuan J-P, Wu C -F, Wang J-H "Fucoxanthin, a marine carotenoid present in brown seaweeds and diatoms: Metabolism and bioactivities relevant to human health," J. Marine Drugs, 9, 1806-1828, 2011

[22] Cho S-Y, Miyashita K, Miyazawa T, Fujimoto K, Kaneda T "Autoxidation of ethyl eicosapentaenoate and docosahexaenoate," J. American Oil Chemists' Soci., 64, 876-879, 1987.

[23] Miyashita K, Takagi T "Study on the oxidative rate and prooxidant activity of free fatty acids," J. American Oil Chemists' Soci., 63, 1380-1384, 1986.

[24] Kim M-K, Dubacq J-P, Thomas J-C, Giraud G "Seasonal variations of triacylglycerols and fatty acids in Fucus serratus," J. Phytochem., 43, 4955, 1996.

[25] Meloni D. A, Oliva M. A, Martinez C. A \& Cambraia J "Photosynthesis and activity of superoxide dismutase, peroxidase and glutathione reductase in cotton under salt stress," J. Enviro., Exp., Botany, 49, 69-79, 2003.

[26] $\mathrm{Nu}^{\prime} \mathrm{n}^{\sim}$ ez M , Mazzafera P, Mazorra L. M , Siquira W. J, Zullo M. A. T "Influence of a brassinosteroid analogue on antioxidant enzymes in rice grown in culture medium with $\mathrm{NaCl}$," J. Biologia Plantarum, 47, 67-70, 2003.

[27] Yamaguchi T, Sugimura R, Shimajiri J, Suda M, Abe M, Hosokawa M, Miyashita K "Oxidative stability of glyceroglycolipids containing polyunsaturated fatty acids,” J. Oleo Sci., 61, 505-513, 2012.

[28] Bishov S. J, Henick A. S, Koch R. B "Oxidation of fat in model systems related to dehydrated foods. II. Composition and position of dispersed lipid components and their effect on oxidation rates," J. Food Sci., 26, 198-203, 1961.

[29] Hosokawa M, Miyashita T, Nishikawa S, Emi S, Tsukui T, Beppu F, Okada T, Miyashita K “Fucoxanthin regulates adipocytokine mRNA expression in white adipose tissue of diabetic/obese KK-Ay mice," J. Arch. Biochem. Biophy., 504(1),17-25, 2010. 